\title{
Les monstres invisibles \\ ou \\ Fillette et phallus
}

\author{
Christine Palmiéri
}

«La peur est au centre de mon œuvre »

Seule, jamais vraiment très seule. Sur fond blanc. D'un monde qu'éclaircit son regard, sombre. Qu'occupe tout son corps. Et ceux des personnes aimées qui l'entourent. Jamais seule. Un ourson, un chien protecteur à cajoler. Un essaim d'amies, de sœurs, de mères, de poupées affectueuses devant l'infini. Devant la mort. Devant laquelle elle se dédouble, sort de soi, pour hurler à l'injustice, joue, déjoue le réel avec une complice, une âme-sœur. Intuitive, sous un masque effronté, avec, pour toute arme, l'insolence. Qui étouffe, anesthésie sa sensibilité exacerbée, sa peur. Seule au fond de cette carapace multicouche, elle se bat. Affronte le monde. Manipule les forces négatives. Se fraye un chemin. Crée son propre espace où elle laisse vagabonder son imaginaire entre chiens et loups, taquinerie et tendresse. Elle invente les règles de son propre jeu, ses rôles: séductrice malicieuse, gamine contestataire, femme craintive, mère attentionnée. Voici tracés les paramètres du théâtre fictionnel mais non moins réel de l'univers dans lequel évoluent les petites filles. Celles de Kim Dawn, d'Ève K. Tremblay et d'Élisabeth Eudes-Pascal, femmes-artistes.

Nostalgie, deuil, séduction, combat, lutte semblent vibrer au cœur des ceuvres pourtant allégées par la spontanéité du geste pictural, ou par les expressions ébahies des modèles, leur octroyant une certaine fraîcheur, une candeur envoûtante. En effet c'est selon ces modalités que l'on retrouve d'ailleurs dans les dessins d'enfant en général ou dans les portraits que l'on fait d'eux que les trois artistes expriment une certaine énergie juvénile - spontanéité, vivacité.

Le trait franc, direct et adroit des dessins de Kim Dawn communique sans équivoque le droit de parole des petites filles qu'elle représente. Droit de parole qui impose une écoute sérieuse. Car on n'a pas affaire ici à des protestations enfantines mais à des requêtes qui surprennent, qui en disent long sur leurs états d'âme, sur leur condition même de petites filles. Gestes spontanés et rapides, ces dessins tombent sur la feuille de papier comme un poing lourd, chargé de haine et de colère. «I deserve respect » et «I want to forget », affirmations qui peuvent se passer de commentaires en laissant sous-entendre un drame. Que cherche-t-elle à oublier? Et en même temps pourquoi demande t-elle à être respectée? L'équation semble facile à résoudre, surtout en des temps où les médias nous informent incessamment des revers des êtres humains, de certains êtres humains qui jusquelà paraissaient très en marge de la société mais dont les statistiques nous montrent que leur nombre est de plus en plus inquiétant. Les petites filles de Kim Dawn, dont certaines semblent avoir perdu leurs mains à force de prier ou de frapper du 
poing, semblent surtout avoir perdu confiance, avoir perdu leur ingénuité, leur innocence, peut-être même leur virginité devant ce côté sombre du monde où rodent ces monstres invisibles que sont les pédophiles. Car, sans les nommer, les petites filles de Kim Dawn osent, contrairement à d'autres, revendiquer un peu de dignité, à demi-mots, menaçant toutefois de se dédoubler, elles aussi étirant leur long et fin cou dans une expression qui tient lieu d'insulte: «banana split » voilà ce que je deviendrai semble-t-elle dire, en attendant elle retrouve un peu de chaleur humaine en la compagnie d'un être fidèle et affectueux, un chien, un animal, un vrai. C'est donc par quelques coups de feutre violents que l'artiste imprime noir sur blanc le danger que frôle régulièrement les petites filles, à vivre dans un monde où l'ignominie règne dans les soubassements de l'instinct, où le mal se conjugue à la nécessité de survivre. Où la transgression devient synonyme de désir et de plaisir en enfreignant les lois humaines et peut-être même animales qui sont de protéger sa progéniture, de la respecter, de l'aimer. Ces dessins s'adressent autant à ceux qui enfreignent la loi qu'à ceux qui ne la font pas respecter. Ces dessins, taches noires cinglantes sur la page blanche du silence dégagent malgré tout, par leur trait effronté, une fraîcheur enfantine, signe d'espoir devant l'avenir et c'est en cela que cette production est puissante. Ravivant ces questions souvent refoulées, elle nous secoue dans notre apathie.

C'est par un geste plus fébrile qu'Élisabeth Eudes-Pascal exprime la vulnérabilité et la sensibilité de ces petites filles. Dans un enchevêtrement de traits colorés au pastel. Mauve/deuil, rouge/sang, bleu/solitude pris dans les mailles de lignes noires qui tracent des rituels étranges où, mères, filles et amies semblent être réunies autour d'un même mystère. Dans une intimité féminine, elles semblent se livrer à des cérémonies secrètes en quête des puissances redoutables qui se cachent au fond d'une caverne, dans les branches d'un arbre ancestral? Elles s'adonnent à des transes pour trouver la délivrance, se libérer des angoisses? Le tremblé des lignes exprime l'incertitude, l'inquiétude devant les forces maléfiques, devant la culpabilité, devant le secret qui les lie. Elles semblent scander en chœur: «I want to forget » et « I deserve respect ». Ensemble, elles se prémunissent contre le sort des femmes dans une sorte de rituel initiatique. C'est dans le flou, le brouillage des lignes et des taches que les silhouettes, ombres mnésiques de vestales, hantent le papier, la conscience - maculés de souffrance, de douleur. Prises en faute, les petites filles tournent parfois en dérision la question même du péché originel. Et, dans un même esprit moqueur, elles trouvent refuge dans le modèle de la vie familiale classique, rêve ou utopie, que l'artiste trace dans un emboîtement bédéiste qui n'est pas s'en rappeler le labyrinthe d'une vie avec ses jeux spéculaires, le labyrinthe du logis ou la reine finit par se perdre.

Et c'est par des jeux insolites que les petites filles, ou jeunes adolescentes, des photographies d'Ève K. Tremblay, déjouent l'inquiétude, l'angoisse d'être enfermées derrière les hauts murs d'un pensionnat, à l'abri des regards dangereux du monde. Uniformes déboutonnés, elles s'adonnent à des rites ludiques mettant en scène des signes et des codes qui leur sont propres mais font référence symboliquement, non pas à des querelles anodines, mais à des deuils face à une enfance qui s'étiole: jupon qu'on tire d'un bord à l'autre, visage au regard inquisi- 
teur, larmes coulant le long des joues. Plus vulnérables, cette fois, mais toujours complices, le torse à demi-nu, elles deviennent actrices de leur propre peur en évoquant vraisemblablement une scène devenue un classique du cinéma d'horreur: la scène de la douche du film Psychose d'Hitchcock ou encore la scène non moins connue de Suzanne effrayée, épiée par les vieillards. C'est au cour d'un amoncellement de poupées, les yeux bandés pour la plus grande, qu'elles trouvent refuge, troublées qu'elles sont par leur identité mouvante comme pour conserver les derniers relents d'innocence qui leur restent devant le monde d'adultes qui les attend de l'autre côté du mur. Toujours en quête de liberté, mais cependant craintive, c'est dans un rayon de soleil qu'elles se déchargent de leurs émotions, dont la complexité sème en elle un doute, au point de les pousser à désirer et de refuser en même temps leur devenir-femme incontournable. C'est donc par un travail de mise en scène quasi-cinématographique que les photos d'Ève K. Tremblay semblent croquer sur le vif ces scènes intimistes de jeux, laissant émaner de façon remarquable la fraîcheur ingénue des modèles. Tout comme Kim Dawn et Élisabeth Eudes-Pascal, elle nous rend voyeur/se des affects qu'éprouvent les petites filles exposées aux travers d'un monde de plus en plus égoïste, inconscient et sans culpabilité, à l'aube de leur maturité. D'emblée elles se victimisent, manifestent le « mal-être ». de leur existence, de leur condition au sein d'un monde d'adulte qui ne verrait en elles qu'un corps conçu comme lieu de désir et de jouissance. Pascal Quignard ne dit-il pas «L'homme est un regard désirant qui cherche une autre image derrière tout ce qu'il voit $»$. Sorte d'image originelle qui l'obsède. Il raconte aussi «Il se trouve une pierre où est sculpté un facinus grossier que le statuaire a entouré de ces mots: Ici réside le bonheur ». Sans vraiment connaître la source de leur tourment, quelque chose du fond de leur conscience lance un avertissement. Elles vivent dans le doute. Ont des pensées prémonitoires, floues qui les insécurisent. Elles se protègent contre qui? Quoi? Mais elles doivent évacuer leurs craintes. Déjà Louise Bourgeois exorcisant ses angoisses réalisait, en 1968, une œuvre intitulée Fillette représentant un phallus mesurant $51 \mathrm{~cm}$ de haut - sorte d'avorton difforme, tuméfié par la jouissance et effroyable par son format - arme qu'elle inventa pour combattre et tuer les spectres qui menaçaient sa conscience; elle disait: « dans la vie je suis une victime, en art je suis un assassin », en avouant du même coup que « la peur est au centre de son cuvre ».

Si les œuvres de ces trois artistes s'inscrivent dans une esthétique de la candeur, elles manifestent au-delà d'une fausse naïveté la conscience très aiguisée des petites filles qui se protègent contre l'agressivité et la perversité dont elles sont souvent la cible. Elles se permettent d'en jouer, dans une sorte de perversité naïve, inconsciente. C'est ce qui crée l'ambivalence de cette figure qu'est la femmeenfant, qui par nécessité d'autoprotection devient provocatrice ou, à l'inverse, séductrice, jeux dans lequel elle devient très habile, comme par seconde nature. Développant des armes contre l'autorité et le pouvoir masculin, le destin de la petite fille se dessinera dans une tension entre deux pôles qui donnent lieu à deux figures ou clichés, celui de la femme frivole, hyper-séductrice, et celui de la femme machiste, hyper-féministe. Clichés que l'on retrouve dans maintes productions littéraires, cinématographiques et visuelles pour la plupart masculines. 
Ainsi les artistes réunies dans ce dossier prêtent un visage humain à leurs dessins ou modèles, délibérément ou intuitivement, les sortant du carcan représentationnel des figures de Lolita ou d'Alice telles que tracées par le discours masculin. En cela elles « échappent au langage à l'intérieur duquel l'homme les enferme » et révèlent « l'importance pour elles d'établir leur propre rapport au sacré » comme Luce Irigaray l'énonce.

Ainsi les représentations de petites filles réunies à travers ces œuvres dévoilent la face cachée de l'icône angélique ou diabolique des représentations habituelles. L'attracteur qui motive ces productions semble se cristalliser autour d'une émotion qui est celle de la peur, de l'angoisse, non plus devant l'inconnu qui a motivé un nombre impressionnant d'œuvres pour ne pas dire l'art depuis ses origines, mais devant l'inconnu bien concret, qui gît au fond de certains êtres, fait de mains, de bras, de jambes, de sexes, de pulsions lubriques voire même meurtrières, mais dépourvu de tête et surtout de cœur. Ces œuvres nous entraînent dans le vertige de la peur, dans le tourbillon de cérémonies secrètes, dans les méandres de l'intuition, des prémonitions, devant des temps douteux, des individus incapables de voir ou, pire, ne voulant pas croire à ce qui se déroule sous leurs yeux. N'est-ce pas là le grand paradoxe de l'humanité qui par ailleurs croit ou a cru pendant longtemps à ce qu'elle n'a jamais vu? Qui doute plus de ses capacités perceptives que de son potentiel imaginatif? L'enfermement derrière des murs, derrière un tchador ou sous une burga ne suffit pas, ne résout rien et peut-être même excite le mal. Pour Naddeije Laneyrie-Dagen, « le corps est le lieu des dernières incertitudes et des plus folles angoisses $»$.

Ainsi le secret hallucinant qui lie souvent l'agresseur et l'agressée plonge la victime dans une confusion extrême, dans un cycle de culpabilité qui la pousse à des supplications du genre: "I want to forget », dans le silence de la page blanche qui se referme après chaque épisode. Ces œuvres réalisées dans le trouble, l'incertitude, l'infini, semblent s'appuyer comme chez Louise Bourgeois sur un savoir abyssal et, comme elle, les artistes présentées ici semblent dire « j'ai confiance dans l'inconscient $\gg$.

Ces œuvres à l'apparente innocence dénotent un processus énonciateur puissant révélateur d'une phénoménologie en action qui par l'entremise de quelques signes nous emporte par empathie dans les drames humains que l'humanité même ne peut se défaire, ne peut éradiquer malgré la honte qu'ils font porter à nos sociétés dites civilisées. «I deserve respect » murmurent à l'unisson les petites filles. 